\title{
Binding of the basic fibroblast growth factor (bFGF) by soluble components of human umbilical $\operatorname{cord}^{\text {t }}$
}

\author{
Krzysztof Sobolewski ${ }^{1}$, Edward Bańkowski ${ }^{1 凶}$, Jerzy Pałka $^{1}$ and Stefan Jaworski ${ }^{2}$ \\ ${ }^{1}$ Department of Medical Biochemistry, and ${ }^{2}$ Department of Gynaecology, Medical Academy of \\ Biatystok, Biatystok, Poland
}

Received: 13 November, 2001; revised: 19 July, 2002; accepted: 12 November, 2002

Key words: EPH-gestosis, fibroblast growth factor, pre-eclampsia, Wharton's jelly, umbilical cord artery

\begin{abstract}
Pre-eclampsia, the most common pregnancy associated syndrome, is connected with remodelling of extracellular matrix of the umbilical cord tissues. Since the fibroblast growth factor (FGF) is known to be a stimulator of collagen and glycosaminoglycan biosynthesis, one may expect that it plays an important role in such a remodelling. Studies performed on the umbilical cords of 10 control and 10 pre-eclamptic newborns demonstrated that both the umbilical cord arterial wall and Wharton's jelly contain FGF mainly in complexes with the components of different molecular mass. Pre-eclampsia is associated with a decrease of endogenous FGF-binding by soluble high molecular mass components of the umbilical cord. It is suggested that FGF released from these complexes may be actively bound by fibroblasts of the umbilical cord, stimulating them to produce collagen and sulphated glycosaminoglycans.
\end{abstract}

It is known that the extracellular matrix (ECM) is the place where growth factors, like fibroblast growth factor (FGF), transforming growth factor- $\beta$ (TGF- $\beta$ ) and insulin-like growth factors (IGFs) are concentrated and stabilised in the vicinity of cells. The release of these factors from matrix storage may in turn control cell growth and differentiation and stimulate the cells to produce the extracellular matrix components. This suggests that both binding and storage of growth factors by the matrix are important mechanisms in the control of cellular metabolism (Taipale \& Keski-Oja, 1997; Hardingham \& Fosang, 1992; Jackson et al., 1991; Ruoslahti \& Yamaguchi, 1991).

Pre-eclampsia (EPH-gestosis) is the most common pathological syndrome observed in

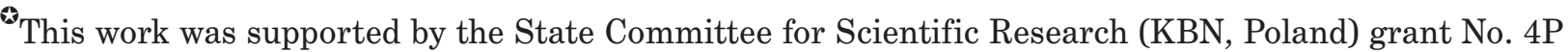
$05 \mathrm{E} 07715$.

${ }^{\otimes}$ Corresponding author: Edward Bańkowski, Department of Medical Biochemistry, Medical Academy of Białystok, A. Mickiewicza 2, 15-230 Białystok-8, Poland; tel.: (48 85) 748 5577; fax: (48 85) 745 616; e-mail: edward@amb.edu.pl
}

Abbreviations: bFGF, basic fibroblast growth factor; GAGs, glycosaminoglycans; IGF-I, insulin-like growth factor-I; UCA, umbilical cord artery. 
pregnancy. It is associated with a significant increase in collagen content in the umbilical cord arteries (UCAs) (Bańkowski et al., 1993; 1999; Romanowicz, 1998) and an early replacement of hyaluronic acid by sulphated glycosaminoglycans (GAGs) both in UCA and in Wharton's jelly (Sobolewski et al., 1997). Since some of these factors (IGF-I, FGF) are known to stimulate collagen and GAGs biosynthesis, one may expect that they play an important role in remodelling of the umbilical cord tissues (Zhang \& Phan, 1996; Feld et al., 1995; Thomas, 1987).

It has been reported in our recent papers that both the UCA and Wharton's jelly contain IGF-binding proteins and that their amount and the binding activities become altered in pre-eclampsia (Pałka et al., 2000). On the other hand, it is known that sulphated GAGs, especially heparin sulphate and heparin in proteoglycans bind FGF (Hardingham \& Fosang, 1992; Jackson et al., 1991; Ruoslahti \& Yamaguchi, 1991). Binding of bFGF by the umbilical cord tissues has not been investigated so far. Therefore binding of this factor by UCA and Wharton's jelly was evaluated in new borns from normal pregnancy and pre-eclampsia.

\section{MATERIALS AND METHODS}

Tissue material. Umbilical cords of 10 control and 10 pre-eclamptic newborns, delivered between 38 and 41 weeks of gestation. The control material was taken from newborns delivered by healthy mothers, aged $23-35$, with normal blood pressure (systolic 100-139 mm $\mathrm{Hg}$, diastolic 70-89 mm Hg). The mothers presented no symptoms of edema or renal failure. The mean body mass of newborns was $3.668 \pm 371 \mathrm{~g}$. The pre-eclamptic newborns were delivered by mothers, aged 22-34, who demonstrated an elevation of blood pressure (systolic > $140 \mathrm{~mm} \mathrm{Hg}$, diastolic > $90 \mathrm{~mm} \mathrm{Hg}$ ), proteinuria (> $500 \mathrm{mg} / \mathrm{l}$ ) and edema after bed rest. The patients with cardio-vascular, renal and metabolic diseases were excluded. The mean body mass of newborns was $3056 \pm 498$ g. In all cases, $20 \mathrm{~cm}$ long sections of the umbilical cords were excised beginning from their placental end. The UCAs and Wharton's jelly were carefully separated.

Preparation of tissue extracts. The tissues (200 mg) were homogenised in $2 \mathrm{ml}$ of $1 \mathrm{M}$ acetic acid (acidic extract) or in $0.05 \mathrm{M}$ Tris $/ \mathrm{HCl}$, pH 7.6 (neutral extract) with the use of a mechanical homogenizer, and then submitted to ultrasonification $\left(20 \mathrm{kHz}, 3 \times 10 \mathrm{~s}\right.$, at $\left.0^{\circ} \mathrm{C}\right)$. The homogenates were centrifuged at 10000 $\times \boldsymbol{g}$ for $10 \mathrm{~min}$ at $4^{\circ} \mathrm{C}$. The supernatants were collected and evaporated in a vacuum concentrator (Labconco).

Sodium dodecyl sulphate/polyacrylamide gel electrophoresis (SDS/PAGE). Slab SDS/PAGE was performed according to the method of Laemmli (1970) in 10\% polyacrylamide gel. The dried materials were dissolved in $0.5 \mathrm{ml}$ of sample buffer $(0.0625 \mathrm{M}$ Tris/HCl, pH 6.8, containing 20\% glycerol and $5 \%$ SDS), and $20 \mu \mathrm{l}$ of each were applied to the gel. The following Bio-Rad molecular mass standards were used: $139.9 \mathrm{kDa}, 86.8 \mathrm{kDa}$, $47.8 \mathrm{kDa}, 33.3 \mathrm{kDa}, 28.6 \mathrm{kDa}$ and $20.7 \mathrm{kDa}$. After SDS/PAGE, the gels were allowed to equilibrate in a mixture of $0.025 \mathrm{M}$ Tris and $0.2 \mathrm{M}$ glycine in $20 \%(\mathrm{v} / \mathrm{v})$ methanol for $5 \mathrm{~min}$. The protein was transferred to $0.2 \mu \mathrm{m}$ pore-sized nitrocellulose, at $100 \mathrm{~mA}$ for $1 \mathrm{~h}$ using a Sigma-Aldrich SV 20-SDB electrophoresis unit. Nitrocellulose was blocked with $3 \%$ IGEPAL CA-630 in TBS for $30 \mathrm{~min}$, then in 1\% bovine serum albumin (BSA) in TBS for $2 \mathrm{~h}$ and finally in $0.1 \%$ Tween 20 in TBS for 10 min at room temperature, and submitted to ligand binding (Hosenlopp et al., 1986).

\section{Ligand blotting (125I-FGF binding).}

FGF-binding by the components of tissue extract on the membrane was carried out as described by Hosenlopp et al. (1986) with $1 \mu \mathrm{Ci}$ of ${ }^{125} \mathrm{I}$-bFGF (Sigma) in $10 \mathrm{ml}$ of mixture containing $1 \%$ BSA and $0.1 \%$ Tween 20 in TBS. The membrane was washed twice with $20 \mathrm{ml}$ of $0.1 \%$ Tween 20 in TBS, 3 times with $20 \mathrm{ml}$ 
of TBS, dried for $1 \mathrm{~h}$ and exposed to Kodak $\mathrm{X}$-Omat film for 3 days at $-70^{\circ} \mathrm{C}$.

Detection of bFGF in the extracts of the UCA-wall and Wharton's jelly. The extracts were submitted to SDS/PAGE and ligand blotting with the use of monoclonal anti-human bFGF, Clone FB-8 (Sigma) (Hosenlopp et al., 1986).

All the experiments were performed on 20 tissue samples, taken from 10 control and 10 pre-eclamptic subjects. Representative electrophoregrams are presented.

\section{RESULTS}

Figure 1A shows the position of endogenous bFGF in acetic acid extracts from UCA and

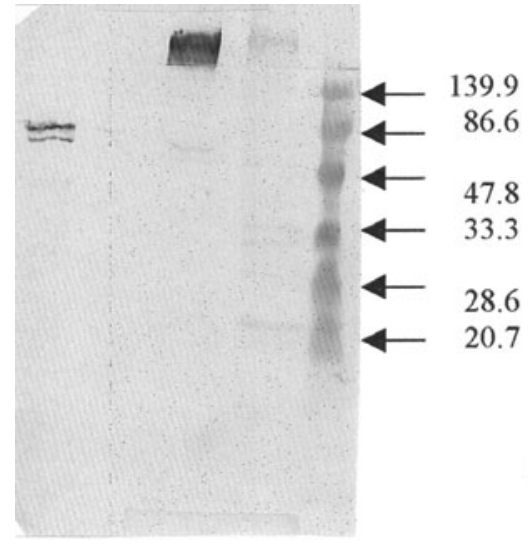

$\begin{array}{llll}1 & 2 & 3 & 4\end{array}$

A slight amount of endogenous FGF is bound to this component of pre-eclamptic Wharton's jelly. Only trace amounts of endogenous FGF are bound to the components of lower molecular mass (lane 4).

The neutral extract (Fig. 1B) from control UCA contains more endogenous bFGF than does the acidic extract. This factor forms complexes with the components of different molecular mass. The band of the highest electrophoretic mobility corresponds to the molecular mass of free FGF, about $25 \mathrm{kDa}$ (lane 1). No significant differences between control (lane 1) and pre-eclamptic (lane 2) UCA were found. Less endogenous FGF was extracted from both control (lane 3) and pre-eclamptic (lane 4) Wharton's jelly than from UCA. It seems that in this tissue a part of FGF is

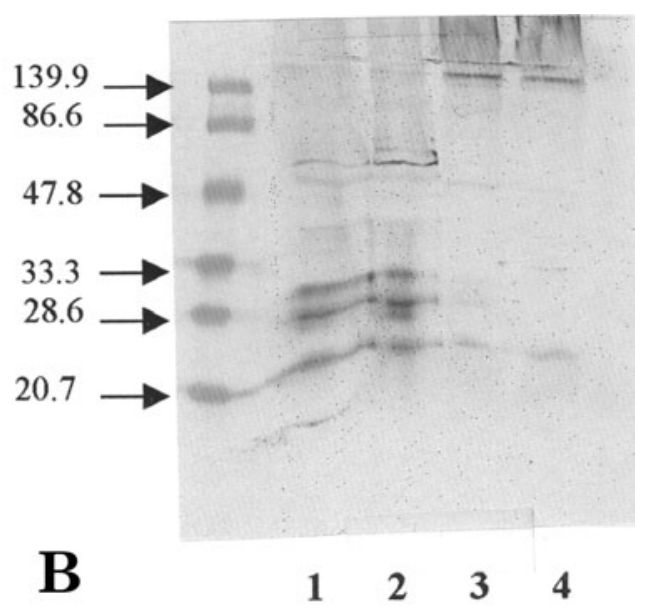

Figure 1. The SDS/PAGE pattern of endogenous fibroblast growth factor (FGF) on acidic (A) and neutral (B) extracts of umbilical cord artery (UCA) and Wharton's jelly.

The ligand blotting procedure was performed as described in Materials and Methods. Control UCA (lane 1), pre-eclamptic UCA (lane 2), control Wharton's jelly (lane 3), pre-eclamptic Wharton's jelly (lane 4).

Wharton's jelly. In control UCA two closely located bands of about $85 \mathrm{kDa}$ are apparent (lane 1). Only a trace amount of this material was detected in pre-eclamptic material (lane 2). Also significant differences between UCA and Wharton's jelly are evident. The control Wharton's jelly contains a large amount of FGF bound to a high molecular mass compound (lane 3), which does not penetrate the separating gel. In contrast to control, only a bound to high molecular mass component(s), which hardly penetrated the separating gel and only a part (a trace amount) corresponds to free FGF (lanes 3 and 4).

It can be seen from Fig. 2A that the acetic acid extract from the control UCA wall contains at least two additional constituents, besides those bound to endogenous bFGF, which are able to bind exogenous, radioactive FGF. One of them $(30 \mathrm{kDa})$ is distinctly visible and 

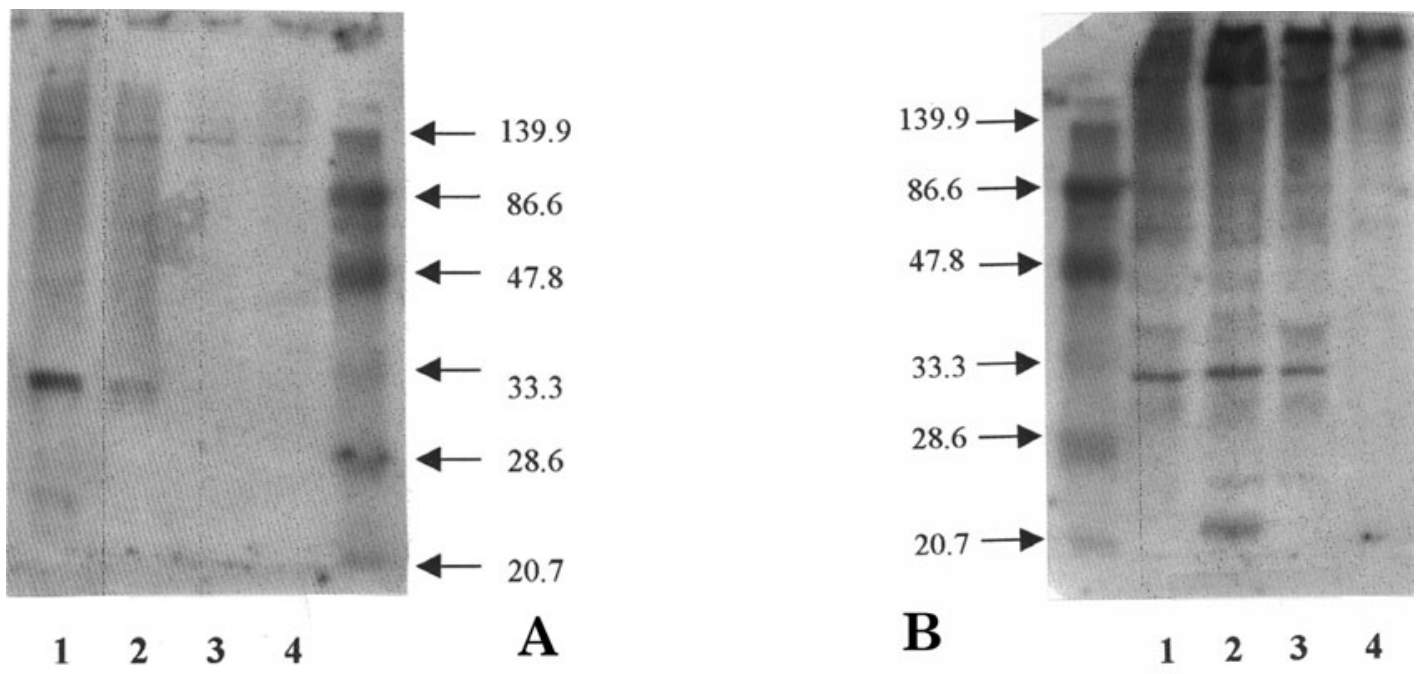

Figure 2. The SDS/PAGE pattern of FGF-binding compounds of acidic (A) and neutral (B) extracts of umbilical cord artery (UCA) and Wharton's jelly submitted to the ligand blotting procedure.

Control UCA (lane 1), pre-eclamptic UCA (lane 2), control Wharton's jelly (lane 3), pre-eclamptic Wharton's jelly (lane 4).

the other (135 kDa) in less intensive (lane 1). In pre-eclamptic UCA (lane 2) the $30 \mathrm{kDa}$-binding site is less intensively expressed. In contrast to UCA-wall, in the acidic extract from Wharton's jelly binding of exogenous ${ }^{125}$ I-FGF is not observed with the exception of a compound of about $135 \mathrm{kDa}$ (lane 3). Pre-eclampsia does not affect binding of FGF by Wharton's jelly constituents (lane 4).

As can be seen from Fig. 2B the neutral extracts both from the UCA wall and Wharton's jelly contain several additional constituents differing in molecular mass, which bind exogenous radioactive FGF. Some of them are of high molecular mass and do not penetrate the separating gel. In control UCA at least 5 compounds which bind exogenous radioactive FGF have been detected. Their molecular mass varies from $30 \mathrm{kDa}$ to at least $140 \mathrm{kDa}$ (lane 1). All the bands detected in control UCA are apparent also in pre-eclamptic UCA. The amount of ${ }^{125}$ I-FGF-binding component(s) which do not penetrate the separating gel seems be even higher than in control UCA. Furthermore, the extract from pre-eclamptic UCA contains some additional ${ }^{125}$ I-FGF binding compounds, which were not observed in control UCA. In addition to the above men- tioned high molecular mass components, two low molecular mass $(22-26 \mathrm{kDa}){ }^{125} \mathrm{I}-\mathrm{FGF}$ binding compounds are visible at the bottom of the gel (lane 2). ${ }^{125}$ I-FGF-binding by the neutral extracts of control Wharton's jelly (lane 3) is very similar to that exhibited by control UCA extracts. Pre-eclampsia is associated with the disappearance of several (mainly low-molecular) FGF-binding compounds (lane 4).

\section{DISCUSSION}

Extracellular matrix is a place where various growth factors are bound and immobilised (Carey, 1997; Iozzo, 1998). Heparan sulphate and heparin are known as the main FGF-binding components (Jackson et al., 1991; Tanaka et al., 1998). The sulphated proteoglycans protect FGF against proteolysis and allow concentration of significant amounts of this factor in the vicinity of cells (Tanaka et al., 1998; Gallagher, 1996).

It has been found that endogenous bFGF extracted by acetic acid either from the UCA or Wharton's jelly is bound to some components of high molecular mass. Furthermore, these 
extracts contain some FGF-binding constituents, which are not fully saturated with endogenous FGF and are able to bind exogenous ${ }^{125} \mathrm{I}$-FGF.

The neutral extracts from control UCA and Wharton's jelly contain more endogenous FGF associated with the components of different molecular mass able to bind FGF. Furthermore, these extracts seem to contain some free FGF. The sulphate-containing proteoglycans are strongly anionic substances (Jackson et al., 1991; Hardingham \& Fosang, 1992). Their solubility in neutral solutions is distinctly higher than in acidic conditions. This seems be the reason why the amount of proteoglycan-bound bFGF in neutral extracts is higher than in acidic conditions.

It is worthy of note that neutral extracts, similarly as acidic extracts, contain the constituents which are not saturated with endogenous FGF and can bind to exogenous ${ }^{125}$ I-FGF in the experimental conditions.

Our experiments proved that the extracts from UCA or Wharton's jelly contain several soluble FGF-binding compounds. It can not be excluded that the examined tissues contain other FGF-binding components which are not extracted in the experimental conditions. It is known that high molecular mass proteoglycans are extracted by the concentrated solutions of guanidine hydrochloride (Yanagishita et al., 1987). However, it would be impossible to perform the FGF-binding reaction in concentrated guanidine- $\mathrm{HCl}$ since the existing complexes would dissociate. The extractability of FGF-binding compounds depends on the $\mathrm{pH}$ of extracting solution. Distinctly more FGF-binding material has been extracted by neutral buffer than by acidic solution.

It is of interest that the high molecular mass components extracted with acidic solutions either from the UCA wall or Wharton's jelly of pre-eclamptic newborns contain less bound endogenous FGF than controls. Such differences were not observed in neutral extracts.
Basing on the presented results one may conclude that pre-eclampsia is associated with a decrease of endogenous FGF-binding by high molecular mass components of the umbilical cord. Also it may be suggested that free FGF (released from high molecular mass complexes) can be actively bound by fibroblasts of the umbilical cord, stimulating them to produce collagen and sulphated GAGs.

Various FGF-binding compounds of the UCA wall and Wharton's jelly differ in affinity to FGF and preference for binding endogenous FGF. The binding compounds saturated with endogenous FGF may be in accessible to exogenous FGF.

It is known that the umbilical cord, especially Wharton's jelly, contains a low number of cells (mainly myofibroblasts) and large amounts of collagen, hyaluronic acid and sulphated glycosaminoglycans. These cells must be strongly stimulated to produce such a large amount of extracellular matrix components (Sobolewski et al., 1997; Bańkowski et $a l .$, 1996). Both IGF-I (Pałka et al., 2000) and FGF seem to be responsible for such stimulation. The immobilisation and concentration of these growth factors by binding constituents may be the mechanisms which promote the biosynthesis of extracellular matrix components of the umbilical cord.

\section{R E F E R E N C E S}

Bańkowski E. (1999) Collagen of the umbilical cord and its alteration in EPH-gestosis. Proc Indian Acad Sci (Chem Sci).; 111: 207-13.

Bańkowski E, Romanowicz L, Jaworski S. (1993) Collagen of umbilical cord arteries and its alterations in EPH-gestosis. J Perinat Med.; 21: 491-8.

Bańkowski E, Sobolewski K, Romanowicz L, Chyczewski L, Jaworski S. (1996) Collagen and glycosaminoglycans of Wharton's jelly and their alterations in EPH-gestosis. Eur $J$ Obst Gyn Reprod Biol.; 66: 109-17. 
Bańkowski E, Pawlicka E, Jaworski S. (1997) Collagen biosynthesis in the wall of umbilical cord artery and its alteration in EPH-gestosis. Pathophysiology.; 4: 9-13.

Carey DJ. (1997) Syndecans: multifunctional cell-surface co-receptors. Biochem J.; 327: $1-16$.

Feld SM, Hirschberg R, Artishevsky A, Nast C, Adler SG. (1995) Insulin-like growth factor I induces mesengial proliferation and increases mRNA and secretion of collagen. Kidney Intern.; 48: 45-51.

Gallagher JT. (1996) Heparan sulphate proteoglycans. The control of cell growth. In: Extracellular matrix 2. Comper WD. ed, pp 230-45. Harwood Academic Publishers $\mathrm{GmbH}$, Amsterdam.

Hardingham TE, Fosang AJ. (1992) Proteoglycans: many forms and many functions. FASEB J.; 6: 861-70.

Hosenlopp P, Seurin D, Segovia-Quinso B, Hardouin S, Bionoux M. (1986) Analysis of serum insulin-like growth factor binding proteins using Western blotting: use of the method for titration of the binding proteins and competitive binding studies. Anal Biochem.; 154: 138-43.

Iozzo RV. (1998) Matrix proteoglycans: from molecular design to cellular function. Annu Rev Biochem.; 67: 609-52.

Jackson RL, Busch SJ, Cardin AD. (1991) Glycosaminoglycans: molecular properties, protein interaction, and role in physiological processes. Physiol Rev.; 71: 481-525.

Laemmli UK. (1970) Cleavage of structural proteins during the assembly of the head of bacteriophage T4. Nature.; 227: 680-5.
Pałka J, Bańkowski E, Jaworski S. (2000) An accumulation of IGF-I and IGF-binding proteins in human umbilical cord. Mol Cell Biochem.; 206: 133-9.

Romanowicz L, Bańkowski E, Jaworski S. (1998) Electrophoretic and chromatographic patterns of glycosaminoglycans of the umbilical cord vessels and their alteration in EPH-gestosis. Acta Biochim Polon.; 45: 805-9.

Ruoslahti E, Yamaguchi Y. (1991) Proteoglycans as modulators of growth factor activities. Cell.; 64: 867-96.

Sobolewski K, Bańkowski E, Chyczewski L, Jaworski S. (1997) Collagen and glycosaminoglycans of Wharton's jelly. Biol Neonate.; 71: 11-21.

Taipale J, Keski-Oja J. (1997) Growth factors in the extracellular matrix. FASEB J.; 11: 51-9.

Tanaka Y, Kimata K, Adams DH, Eto S. (1998) Modulation of cytokine function by heparan sulfate proteoglycans: sophisticated models for the regulation of cellular responses to cytokines. Proc Assoc Am Physicians.; 110: 118-25.

Thomas KA. (1987) Fibroblast growth factors. FASEB J.; 1: 434-40.

Yanagishita M, Midura RJ, Hascall VC. (1987) Proteoglycans: isolation and purification from tissue cultures. Methods Enzymol.; 138: 279-89.

Zhang K, Phan SH. (1996) Cytokines and pulmonary fibrosis. Biol Signals.; 5: 232-9. 